\title{
ON DETERMINATION OF MINOR COEFFICIENT IN A PARABOLIC EQUATION OF THE SECOND ORDER
}

\author{
E.I. Safonov \\ Ugra State University, Khanty-Mansyisk, Russian Federation \\ E-mail:dc.gerz.hd@gmail.com
}

\begin{abstract}
An inverse problem of recovering the minor time-dependent coefficient in a parabolic equation of the second order is considered. The unknown coefficient is the controlling parameter. The inverse problem lies in finding the solution of an initial-boundary value problem for this parabolic equation and this timedependent coefficient using data of the initial-boundary value problem and point conditions of overdetermination. Cases of the Dirichlet boundary conditions and oblique derivative conditions are considered. Conditions under which the theorem of existence and solution uniqueness is applicable for the given inverse problem is described; the numerical solution method is described, and its justification is given. All the considerations are carried out in Sobolev spaces. Solution of the direct problem is based on the finite element method and the finite difference method. The proposed algorithm for the numerical solution consists of three stages: initialization of the massive that describes geometry of the area and the boundary vector; implementation of integrative calculation of the desired coefficient using the finite element method; implementation of the finite difference method. Results of numerical experiments are presented, and numerical solution of the model inverse problem is constructed in the case of Neumann boundary conditions; dependency of an error in calculation of the controlling parameter on the variation of the equation coefficients and the noise level of the overdetermination data for domains with different number of nodes that depend on an observation point is described. Results of the calculations show a good convergence of the method. In the case when introduced noise level is $10 \%$, the error between the desired and the obtained solution increases from 8 to 35 times, though the graph of recovered coefficient remains close to the solution graph and repeats its outlines.

Keywords: finite element method; parabolic equation; inverse problem.
\end{abstract}

Introduction

We consider the question of recovering a lower order coefficient in the parabolic equation

$$
u_{t}-A(x, t, D) u+p(t) u=f(x, t),(x, t) \in Q=G \times(0, T) .
$$

where $G$ is a bounded domain in $R^{n}$ with boundary $\Gamma \in C^{2}$ and $A$ is a second order elliptic operator of the form

$$
A(x, t, D) u=\sum_{i, j=1}^{n} a_{i j}(x, t) u_{x_{i} x_{j}}+\sum_{i=1}^{n} a_{i}(x, t) u_{x_{i}}+a_{0}(x, t) u .
$$

The equation (1) is furnished with the boundary and initial condition

$$
u_{t=0}=u_{0}, B u_{S}=g(t, x), S=\Gamma \times(0, T),
$$

where $B u=u$ or $B u=\frac{\partial u}{\partial \gamma}+\sigma(x, t) u$. Here $\gamma=\left(\gamma_{1}(x, t), \ldots, \gamma_{n}(x, t)\right)$ is a smooth nontangent vector field on $S$. The overdetermination condition is written as

$$
u\left(x_{0}, t\right)=\psi(t) .
$$

Thus, the problem can be stated as follows: given functions $\psi, u_{0}, g$, find a solution $u$ to the equation (1) and the function $p(t)$ such that the equalities (1), (2), and (3) hold. The parameter $p$ is actually a control parameter. This inverse problem is a classical problem and numerous examples can be found in [1-6]. The existence and uniqueness theorems of solutions to this inverse problem are exposed in [7-10]. The articles $[7,8]$ contains the conditions of global (in time) solvability of this problem and 
the local solvability theorems can be found in $[9,10]$ and some other articles. At last, the articles [11$20]$ are devoted to numerical calculations of solutions to this problem. The main approach to numerical solving is a reduction of the inverse problem in question to a linear inverse problem by means of the change of variable $u=v \exp \left(-\int_{0}^{t} p(\tau) d \tau\right)$. After the change we arrive at a new inverse problem of recovering the source function of the form $q(t) f(x, t)$ (the function $q=\exp \left(\int_{0}^{t} p(\tau) d \tau\right)$ is an unknown function). The latter problem under the natural conditions on the data is always solvable. However, the inverse change of variables in certain sense is not always possible, since it is not known a priori that the result of recovering, i.e. the function $q$, is positive (in this case we can determine the function $p$ ). The global existence theorems (the most essential results belongs to Prilepko A.I. [7]) rely on the maximum principle and rather rigid conditions for the data. We do not use this change of variables in contrast to other article and this all allows to treat larger classes of the data.

The main numerical methods used in the above-cited articles are the finite difference methods and variational methods. In some cases only some model problems are discussed. In this article we use the theoretical results of the articles $[9,10]$ which are constructive and can serve as the base of a numerical algorithm. The numerical realization relies on the finite element methods. We expose a numerical algorithm and the results of numerical experiments.

In Sect. 1 we present the theoretical justification of the method. Section 2 is devoted to the algorithm of solving the problem. Section 3 contains the description of the numerical realization of the algorithm and the results of numerical experiments are displayed in Section 4.

\section{Basic assumptions and auxiliary results}

We use the Lebesgue spaces $L_{p}(G), L_{p}(Q)$, the Sobolev spaces $W_{p}^{s}(G), W_{p}^{s}(Q)(1 \leq p \leq \infty)$, the Hölder spaces $C^{\beta}(\bar{G})$, and the spaces $L_{p}(0, T ; E)$ with $E$ a Banach space. The latter space consists of strongly measurable functions defined on $(0, T)$ with values in $E$. The definitions of these spaces can be found in [21].

Describe some theoretical results. First, we present the conditions on the data.

Let the symbol $B_{\delta}\left(x_{0}\right)$ stand for the ball centered at $x_{0}$ of radius $\delta$. Denote $Q^{\tau}=(0, \tau) \times G$, and $G_{\delta}=B_{\delta}\left(x_{0}\right)$. A parameter $\delta>0$ is called admissible whenever $\overline{B_{\delta}\left(x_{0}\right)} \subset G$.

The conditions on the coefficients of $A, B$ are as follows:

$$
\begin{gathered}
a_{i j} \in C(\bar{Q}), a_{k} \in L_{p}(Q), \gamma_{i}, \sigma \in C^{1}(\bar{S}), p>n+2 \\
a_{i j} \in L_{\infty}\left(0, T ; W_{p}^{s}\left(G_{\delta}\right)\right), a_{k} \in L_{p}\left(0, T ; W_{p}^{s}\left(G_{\delta}\right)\right), i, j=1,2, \ldots, n, k=0,1, \ldots, n,
\end{gathered}
$$

for some admissible $\delta>0$ and $s>n / p$. We also require that the operator $A$ is elliptic, i. e., there exists a constant $\delta_{0}>0$ such that

$$
\sum_{i, j=1}^{n} a_{i j} \xi_{i} \xi_{j} \geq \delta_{0}\left|\xi^{2}\right| \forall \xi \in R^{n}, \forall(x, t) \in Q
$$

We assume that

$$
u_{0}(x) \in W_{p}^{2-2 / p}(G), g \in W_{p}^{2 k_{0}, k_{0}}(S),\left.B(x, 0) u_{0}(x)\right|_{\Gamma}=g(x, 0) \forall x \in \Gamma,
$$

where $k_{0}=1-1 / 2 p$ in the case of the Dirichlet boundary conditions and $k_{0}=1 / 2-1 / 2 p$ otherwise;

$$
\begin{gathered}
u_{0}(x) \in W_{p}^{2+s-2 / p}\left(G_{\delta}\right) \text { for some admissible } \delta>0 \text { and } s>n / p ; \\
\psi \in W_{p}^{1}(0, T), u_{0}\left(x_{0}\right)=\psi(0) . \\
\left|u_{0}\left(x_{0}\right)\right|>0 .
\end{gathered}
$$

Now we can state the existence theorem [10]. 


\section{Математика}

Theorem 1. Let the conditions (4)-(9) hold. Then, for some number $\tau^{0} \in(0, T]$, on the interval $\left(0, \tau^{0}\right)$ there exists a unique solution $(u, p(t))$ to the problem (1)-(3) such that $u \in L_{p}\left(0, \tau^{0} ; W_{p}^{2}(G)\right)$, $u_{t} \in L_{p}\left(Q^{\tau^{0}}\right), \quad q_{i}(t) \in L_{p}\left(0, \tau^{0}\right), \quad i=1, \ldots, m_{1} . \quad$ Moreover, $\quad u \in L_{p}\left(0, \tau^{0} ; W_{p}^{2+s}\left(G_{\delta_{1}}\right)\right)$, $u_{t} \in L_{p}\left(0, \tau^{0} ; W_{p}^{s}\left(G_{\delta_{1}}\right)\right)$ for all $\delta_{1}<\delta$.

Next, we present some elements of the proof of this theorem. First, we construct an auxiliary function

$$
\Phi_{t}+L_{0} \Phi=f((x, t) \in Q), \Phi_{t=0}=u_{0}(x), B \Phi_{S}=g .
$$

The classical results on solvability of parabolic problems ensure that $\Phi \in W_{p}^{2,1}(Q)$, $\Phi_{t} \in L_{p}\left(0, T ; W_{p}^{s}\left(G_{\delta_{1}}\right)\right), \Phi \in L_{p}\left(0, T ; W_{p}^{2+s}\left(G_{\delta_{1}}\right)\right)$ for all $\delta_{1}<\delta$. We have that $\Phi_{t} \in W_{p}^{s}\left(G ; L_{p}(0, T)\right)$ and thus we can assume that $\Phi_{t} \in C\left(\bar{G} ; L_{p}(0, T)\right)$. The function $w=u-\Phi$, with $u$ a solution to the problem (1)-(3), is a solution to the problem

$$
\begin{gathered}
L w=-p(t) \Phi=F((x, t) \in Q), w_{t=0}=0, B w_{S}=0, \\
w\left(x_{0}, t\right)=\tilde{\psi}(t)=\psi(t)-\Phi\left(x_{0}, t\right) \in W_{p}^{1}(0, T) .
\end{gathered}
$$

Fixing the function $p(t) \in L_{p}(0, \tau)$ and finding a solution $w$ to the problem (11) on the interval $(0, \tau)$, we construct the map $w=w(p)=L^{-1} F$. This map is nonlinear. Taking $x=x_{0}$ in (11), we infer

$$
\tilde{\psi}_{t}-A w\left(x_{0}, t\right)+p(t) \tilde{\psi}=-p(t) \Phi\left(x_{0}, t\right),
$$

which can be written in the form

$$
p(t)=\psi_{0}(t)+R(p), \psi_{0}(t)=\frac{-1}{\psi(t)} \tilde{\psi}_{t}+\frac{1}{\psi(t)} A w\left(x_{0}, t\right),
$$

where the function $w$ is a solution to the direct problem (11). Note that in view of our conditions $\psi(t)>0$ on some segment $\left[0, \tau_{0}\right]$. This equation is actually of the Volterra type.

The following theorem was justified in the proof of Theorem 1 in [8].

Theorem 2. Let the conditions Theorem 1 hold. Then, for some number $\tau^{0} \in(0, T]$, on the interval $\left(0, \tau^{0}\right)$ the operator $R(p): L_{p}\left(0, \tau_{0}\right) \rightarrow L_{p}\left(0, \tau_{0}\right)$ is a contraction and thereby the method of successive approximations $p^{n+1}=\psi_{0}(t)+R\left(p^{n}\right)$ converges as $n \rightarrow \infty$.

\section{Description of the algorithm}

Describe a numerical algorithm. We employ the Neumann boundary condition. In other case the changes in the algorithm are inessential. We take $n=2$ and rewrite the equation (1) and the data in the form

$$
\begin{gathered}
u_{t}-\operatorname{div}(c(x, t) \nabla u)+b(x, t) \nabla u+a(x, t) u+p(t) u=f, \\
b(x, t)=\left(b_{1}(x, t), b_{2}(x, t)\right)^{T}, \nabla u=\left(\frac{\partial u}{\partial x_{1}}, \frac{\partial u}{\partial x_{2}}\right)^{T}, \\
u_{t=0}=u_{0}(x),\left.\frac{\partial u}{\partial n}\right|_{\partial G \times(0, T)}=g,
\end{gathered}
$$

where $n$ is the unit outward normal to $\Gamma$. We also have that

$$
u\left(x_{0}, t\right)=\psi(t) .
$$

The algorithm is iterative and it is based on the finite element method (FEM). 
Given a triangulation of $G$, the nodes of the grid are denoted as $x_{1}, x_{2}, \ldots, x_{m}$. The symbols $\left\{\phi_{i}(x)\right\}$ stand for the corresponding piecewise linear basis functions. Without loss of generality we can assume that the point $x_{0}$ is a mesh node, i. e., $x=x_{j_{0}}$ for some $j_{0}$. We thus have $\phi_{i}\left(x_{j}\right)=\delta_{i j}$, where $\delta_{i j}$ is the Kronecker symbol.

An approximate solution to the problem (15) is written in the form $u_{m}=\sum_{i=1}^{m} c_{i}(t) \varphi_{i}(x)$. It is defined from the system (we integrate (15) over $G$ with the weight $\varphi_{j}$ ).

$$
\left(u_{m t}, \varphi_{j}\right)+\int_{G} c \nabla u_{m} \nabla \varphi_{j} d x+\int_{G}\left(b \nabla u_{m}+a u_{m}+p u_{m}\right) \varphi_{j} d x-\int_{\Gamma} c g(t, x) \varphi_{j} d \Gamma=\int_{G} f \varphi_{j} d x .
$$

This system for the vector-function $\vec{C}(t)=\left(c_{1}(t), c_{2}(t), \ldots, c_{m}(t)\right)$ can be written in the form

$$
M \vec{C}_{t}+K \vec{C}+p M \vec{C}=F+G,
$$

where $M$ and $K$ are matrices with the entries $M_{j i}=\int_{G} \varphi_{i} \varphi_{j} d x$, $K_{j i}=\int_{G}\left(c \nabla \varphi_{i} \nabla \varphi_{j}+b \nabla \varphi_{i} \varphi_{j}+a \varphi_{i} \varphi_{j}\right) d x, \quad F \quad$ and $G$ are vectors with coordinates $\int_{G} f \varphi_{j} d x$ and $\int_{\Gamma} \operatorname{cg} \varphi_{j} d x, \quad i, j=1,2, \ldots, m$, respectively. Let $\vec{U}=\left(u_{k}\left(x_{1}\right), u_{k}\left(x_{2}\right), \ldots, u_{k}\left(x_{m}\right)\right)$, and thus $\vec{U}(0)=\left(u_{0}\left(x_{1}\right), u_{0}\left(x_{2}\right), \ldots, u_{0}\left(x_{m}\right)\right)$. An approximate solution to the system (19) is sought by the finite difference method. We replace (19) with the system

$$
M \frac{\vec{C}_{i}-\vec{C}_{i-1}}{\tau}+K_{i} \vec{C}_{i}+M p \vec{C}_{i}=F_{i}, \vec{C}_{0}=\left.\vec{U}\right|_{t=0}, i=1,2, \ldots, N, \tau=T / N
$$

where $N$ is a positive integer, $K_{i}=K(i \tau)$ and $F_{i}=(F+G)(\tau i)$. Thus, a piecewise constant approximation of a solution $\vec{C}(t)$ to the system (19) is equal to the vector $\vec{C}_{i}$ on the set $((i-1) \tau, i \tau]$. From (17) and the overdetermination condition we have an approximate equality

$$
V_{0} \psi_{t}+\int_{G} c \nabla u_{k} \nabla \varphi_{j_{0}} d x+\int_{G}\left(b \nabla u_{k}+a u_{k}\right) \varphi_{j_{0}} d x+p \psi V_{0}=\int_{G} f \varphi_{j_{0}} d x
$$

where $V_{0}=\int_{G} \varphi_{j_{0}}$. Hence, we obtain that

$$
p=\left(\left(\int_{G} f \varphi_{j_{0}} d x-\int_{G} c \nabla u_{k} \nabla \varphi_{j_{0}} d x-\int_{G}\left(b \nabla u_{k}+a u_{k}\right) \varphi_{j_{0}} d x\right) / V_{0}-\psi_{t}\right) / \psi
$$

The equality (22) is an analog of the equation (14) and is used in successive approximations.

\section{Numerical realization}

Given a triangulation of $G$, we define the nodes $x_{1}, x_{2}, \ldots, x_{m}$ of the grid and construct the corresponding piecewise linear basis functions $\left\{\varphi_{i}(x)\right\}$. Next, we define the quantity $\tau=T / N$ and construct the matrices $M, K$ and the vectors $F_{i}$. We employ the predictor-corrector arguments. An approximation of the function $p(t)$ is a piecewise constant function equal $p_{i}$ on $((i-1) \tau, i \tau)$. Let $p_{0}=\left(f\left(x_{0}, 0\right)+A\left(u_{0}\right)\left(x_{0}, 0\right)-\psi_{t}(0)\right) / \psi(0)$. For simplicity, it is possible to take just $p_{0}=1$. We take also $\vec{C}_{0}=\vec{U}(0)$. Assume that we calculate the vectors $\vec{C}_{1}, \vec{C}_{2}, \ldots, \vec{C}_{l}$ and the constants $p_{1}, p_{2}, \ldots, p_{l}(l<N)$. Put $p_{l+1}^{0}=p_{l}$. Next, we define the quantities $p_{l+1}^{j}(l=1,2, \ldots N)$ from the equality

$$
p_{l+1}^{j+1}=\left[\frac{1}{V_{0}}\left(\left(F_{l+1}\right)_{j_{0}}-\left(K_{l+1} \vec{C}_{l+1}^{j}\right)_{j_{0}}\right)-\psi_{t}((l+1) \tau)\right] / \psi((l+1) \tau),
$$

where the symbol $(\vec{g})_{j_{0}}$ denotes the $j_{0}$-coordinate of the vector $\vec{g}$, and the quantities $\vec{C}_{l+1}^{j}$ are defined as

$$
\vec{C}_{l+1}^{j}=\left(M+\tau\left(K_{l+1}+M p_{l+1}^{j}\right)\right)^{-1}\left(\tau F_{l+1}+M \vec{C}_{l}\right), l=0,1, \ldots, N-1 .
$$




\section{Математика}

This process continues until $\left|p_{l+1}^{j+1}-p_{l+1}^{j}\right|<\varepsilon_{0}$, where $\varepsilon_{0}$ is a prescribed small number. Next, we put $p_{l+1}=p_{l+1}^{j+1}$ and $\vec{C}_{l+1}=C_{l+1}^{j+1}$. As a result, we obtain the vectors $\vec{C}_{1}, \vec{C}_{2}, \ldots, \vec{C}_{N}$ and the constants $p_{1}, p_{2}, \ldots, p_{N}$ which define an approximate solution to our problem for a given triangulation and a parameter $\tau$.

\section{The results of numerical experiments}

The characteristics of the computer used: processor Intel(R) Core(TM) i7-3517U, CPU @ 1,90 GHz 2,40 GHz, RAM 10,00 GB, 64-digital operation system Windows 7 Ultimate.

To simplify the exposition, we present the results of calculation of the function $p(t)$ only. We consider the model problem, where $u=\left(x^{2}+y^{2}+1\right)(1+t), \quad u_{t=0}=x^{2}+y^{2}+1, \quad u_{t}=x^{2}+y^{2}+1$. The domain $G$ coincides with the unit disk centered at $(0,0)$. We consider different points $x_{0}$ as well as different grids (see the fig. 1).

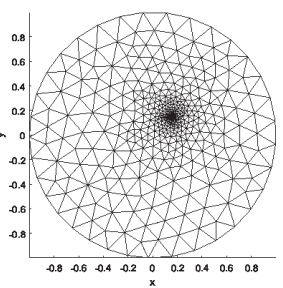

a)

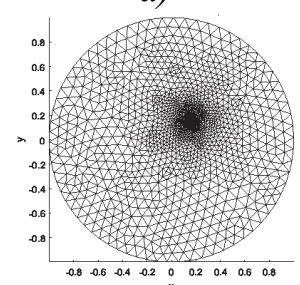

f)

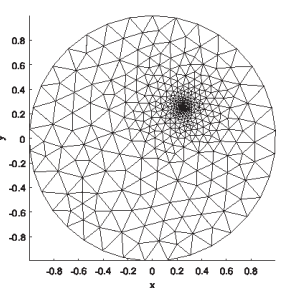

b)

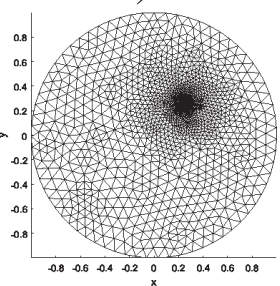

g)

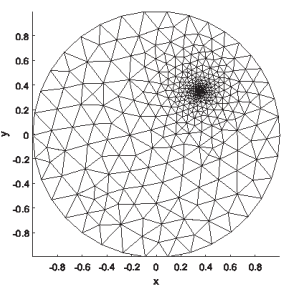

c)

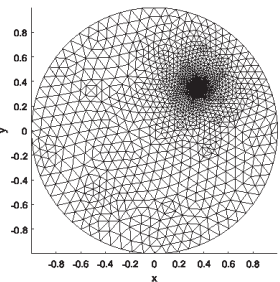

h)

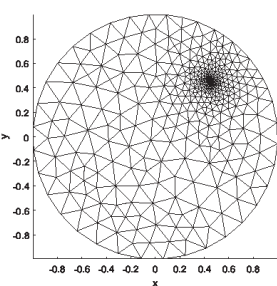

d)

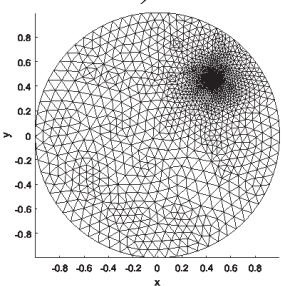

i)

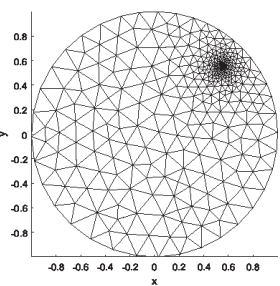

e)

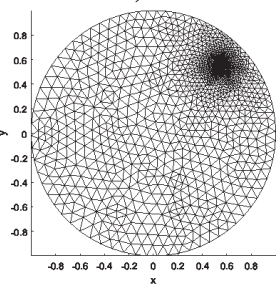

j)

Fig. 1. The grids:

a) $N_{1}=442$; b) $N_{2}=442$; c) $N_{3}=423$; d) $N_{4}=437$; e) $N_{5}=405$;

f) $\left.\left.N_{6}=1731 ; \boldsymbol{g}\right) N_{7}=1731 ; \boldsymbol{h}\right) N_{8}=1653 ;$ i) $\left.N_{9}=1706 ; \boldsymbol{j}\right) N_{10}=1578$

We examine two groups of the data. For the first group, we have $p(t)=(t+1), a=1 /(1+t)$, $b_{1}=x /(2(1+t))$, $b_{2}=y /(2(1+t))$, $c=1 /(1+t)$, $f=2 t+t^{2} x^{2}+t^{2} y^{2}+2 t x^{2}+2 t y^{2}+t^{2}+4 x^{2}+4 y^{2}-1,\left.\quad \frac{\partial u}{\partial n}\right|_{S}=2\left(x^{2}+y^{2}\right)$. Proceed with the results of calculation for the first group of the data for two grids and the data without noise ( $\delta=0$ ) and $\varepsilon_{0}=10^{-5}$ (an error defined by the user). Denote by $\tau_{s}$ the time of calculations in seconds. One more error is the quantity $\bar{\varepsilon}=m_{i} \vec{p}^{k}-\vec{p}(i \tau) \vee \varepsilon=\max _{i}\left|\vec{p}^{k}-\vec{p}(i \tau)\right|$, where $i=1,2, \ldots, N$. We take $T=1$ and $N=100$, $\tau=T / N$ (see the Fig. 2). 


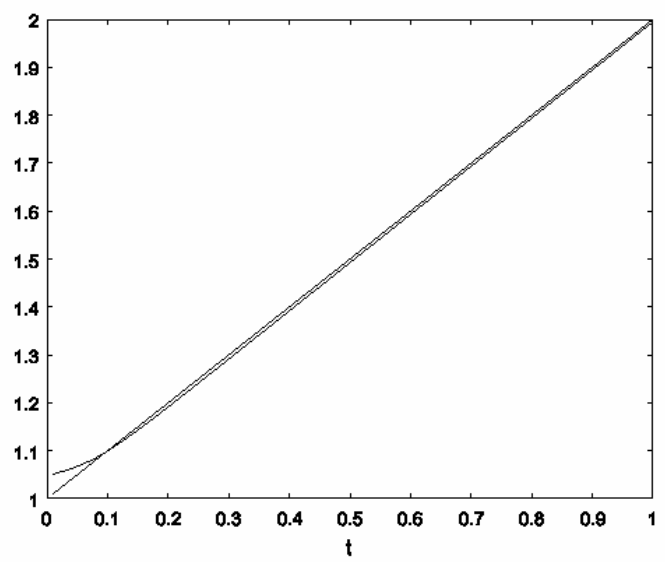

a)

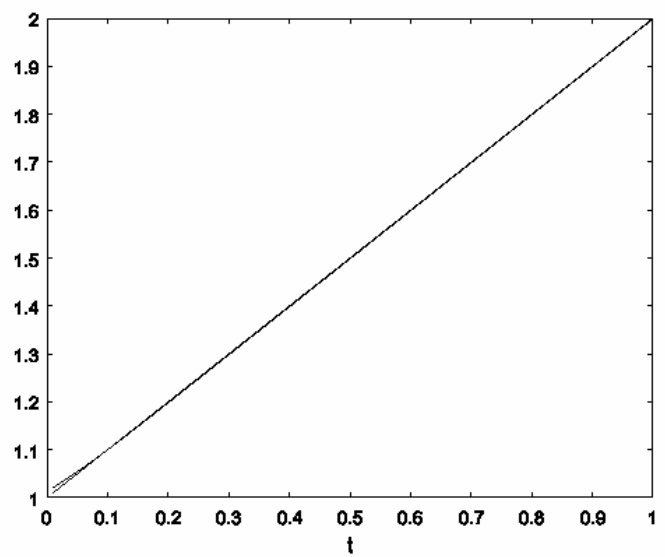

c)

Fig. 2. The results of calculations for the first group of the data:

a) $N_{1}, \varepsilon=0,0415, \tau_{s}=4,37$; b) $N_{5}, \varepsilon=0,024, \tau_{s}=2,81$;

c) $N_{6}, \varepsilon=0,0101, \tau_{s}=190,63$; d) $N_{10}, \varepsilon=0,0058, \tau_{s}=124,85$

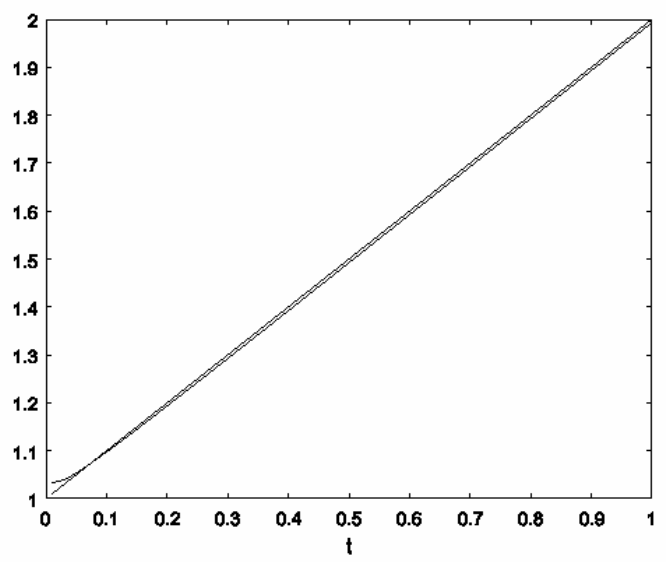

b)

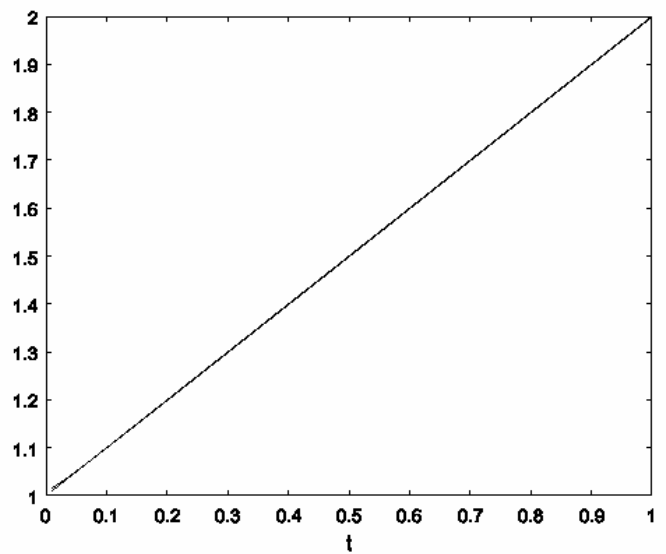

d)

As we can see in Figs. 2, $a-d$, the presented graphs of the functions $p(t)$ and its approximations practically coincide. Next we change the parameters $\varepsilon_{0}$ and $\delta$ to describe the dependence of the error $\varepsilon$ on the level $\delta$ of errors of the data and the parameter $\varepsilon_{0}$. We add the $10 \%$ random noise to the overdetermination data (Fig. 3): $\psi_{i}^{n z}=\psi_{i}(1+\operatorname{random}(1) \cdot 0,2-0,1)$, with random(1) is a random number from $[0 ; 1]$.

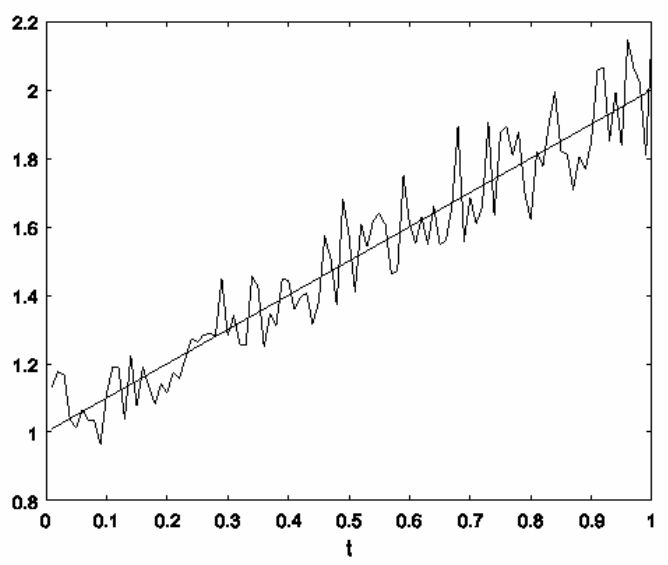

a)

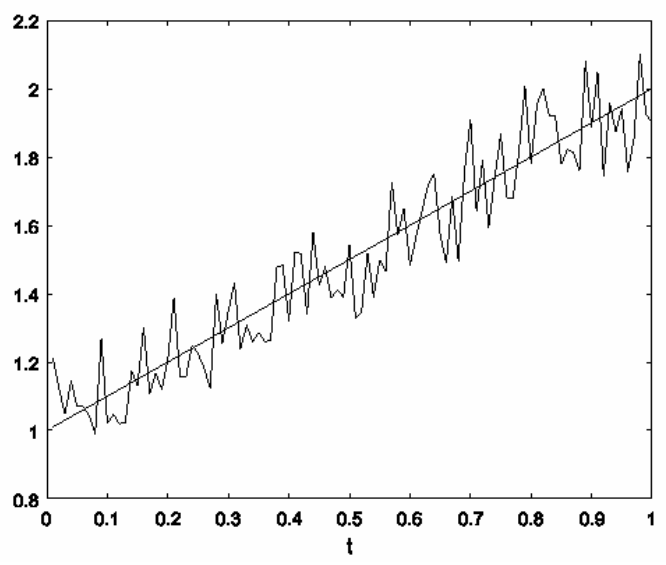

b) 

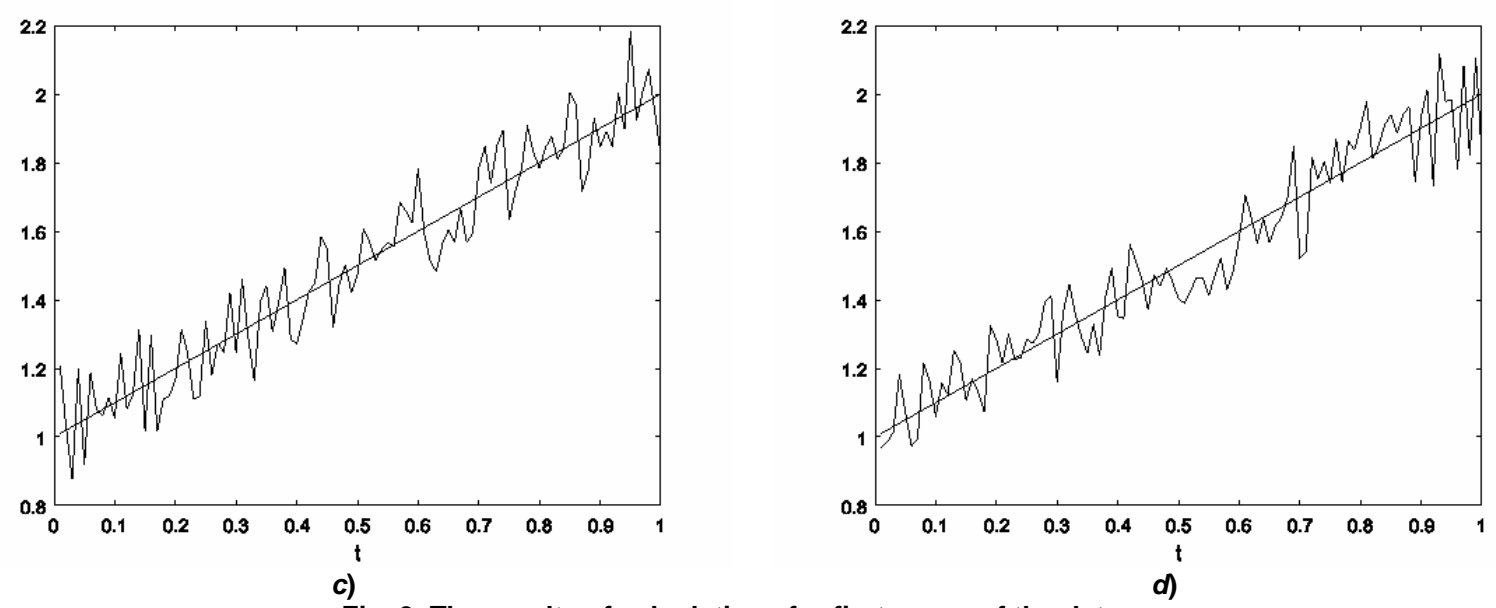

Fig. 3. The results of calculations for first group of the data:

a) $\left.N_{1}, \varepsilon=0,2141, \tau_{s}=5,22 ; \boldsymbol{b}\right) N_{5}, \varepsilon=0,2161, \tau_{s}=4,42$;

c) $N_{6}, \varepsilon=0,2336, \tau_{s}=271,12 ;$ d) $N_{10}, \varepsilon=0,1885, \tau_{s}=122,13$

The results of calculations with a noise are displayed on Fig. 3, $a-d$. The table 1 contains the results of numerical experiments for the first group of the data with fixed $\varepsilon_{0}=10^{-5}$ and different parameters $\delta>0$.

The results of numerical experiments

Table 1

Table 2 for the first group

\begin{tabular}{|c|c|c|c|c|}
\hline No exp. & Grid & $\delta$ & $\varepsilon$ & $\tau$ \\
\hline 1 & $N_{1}$ & 0 & 0,0415 & 4,37 \\
\hline 2 & $N_{1}$ & 0,05 & 0,1033 & 4,85 \\
\hline 3 & $N_{1}$ & 0,1 & 0,2141 & 5,22 \\
\hline 4 & $N_{2}$ & 0,05 & 0,0995 & 4,79 \\
\hline 5 & $N_{2}$ & 0,1 & 0,2163 & 5,02 \\
\hline 6 & $N_{3}$ & 0,05 & 0,1062 & 4,18 \\
\hline 7 & $N_{3}$ & 0,1 & 0,2115 & 4,56 \\
\hline 8 & $N_{4}$ & 0,05 & 0,1039 & 4,08 \\
\hline 9 & $N_{4}$ & 0,1 & 0,2136 & 4,41 \\
\hline 10 & $N_{5}$ & 0 & 0,024 & 2,81 \\
\hline 11 & $N_{5}$ & 0,05 & 0,1213 & 3,34 \\
\hline 12 & $N_{5}$ & 0,1 & 0,2161 & 4,42 \\
\hline
\end{tabular}

The results of calculations for the first group of experiments

\begin{tabular}{|c|c|c|c|c|}
\hline No exp. & Grid & $\delta$ & $\varepsilon$ & $\tau$ \\
\hline 1 & $N_{6}$ & 0 & 0,0101 & 180,37 \\
\hline 2 & $N_{6}$ & 0,05 & 0,1071 & 224,66 \\
\hline 3 & $N_{6}$ & 0,1 & 0,2336 & 271,12 \\
\hline 4 & $N_{7}$ & 0,05 & 0,1057 & 211,27 \\
\hline 5 & $N_{7}$ & 0,1 & 0,2055 & 233,22 \\
\hline 6 & $N_{8}$ & 0,05 & 0,1231 & 180 \\
\hline 7 & $N_{8}$ & 0,1 & 0,2092 & 200,92 \\
\hline 8 & $N_{9}$ & 0,05 & 0,1007 & 163,29 \\
\hline 9 & $N_{9}$ & 0,1 & 0,1993 & 170,97 \\
\hline 10 & $N_{10}$ & 0 & 0,0058 & 124,85 \\
\hline 11 & $N_{10}$ & 0,05 & 0,089 & 119,83 \\
\hline 12 & $N_{10}$ & 0,1 & 0,1885 & 122,13 \\
\hline
\end{tabular}

The error of calculations increases with $\delta$ and the proximity of $x_{0}$ to the center of the circle (see the table 2).

The input data for the second group are as follows: $1 /(t+1), a=\left(t^{x}+1\right), b_{1}=(x+1) \cdot(1+t)$, $b_{2}=\left(x^{2}-2 t\right), c=\left(x^{2}+t\right), f=2 x^{2}-4 x^{2}(t+1)+2 y^{2}-4\left(t+x^{2}\right)(t+1)-2 y\left(2 t-x^{2}\right)(t+1)+2 x$.

Since the error increases when the point $x_{0}$ is closer to the center of the circle, we consider the results in both case with the $10 \%$-noise and without it (Fig. 4). The results are displayed below. 


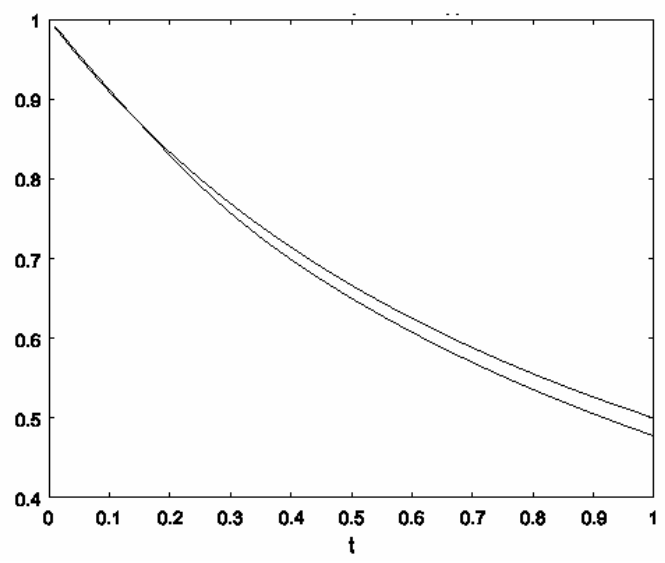

a)

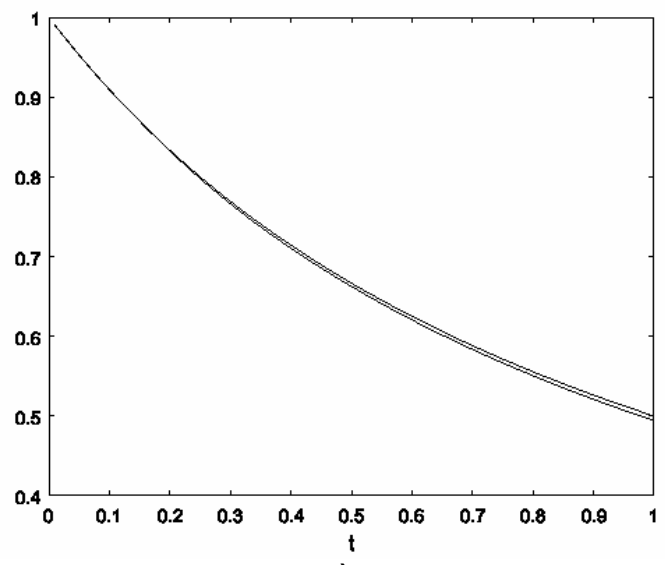

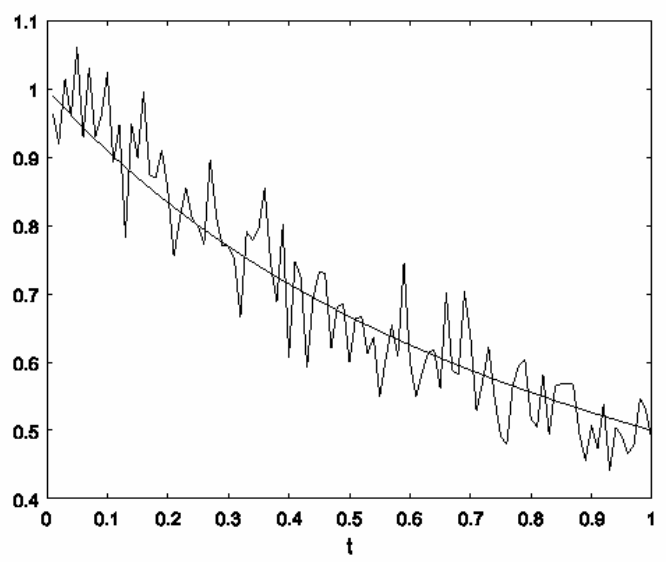

b)

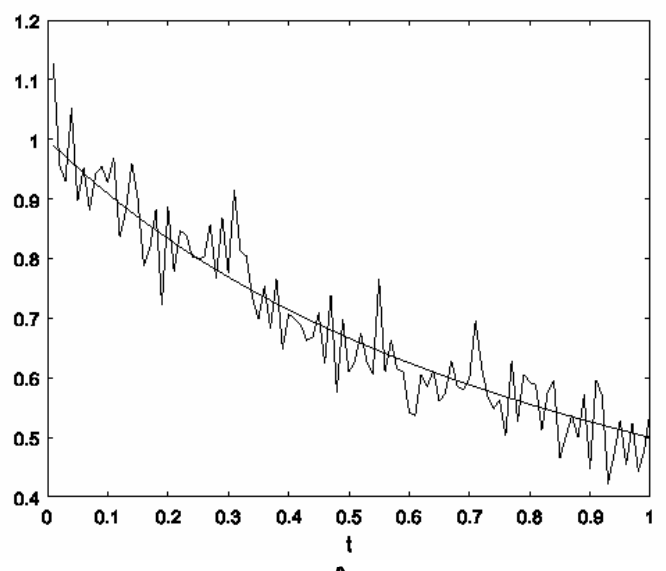

Fig. 4. The results of calculations for the second group of the data:

a) $N_{1}, \varepsilon=0,0224, \tau_{s}=4,51$; b) $N_{1}, \varepsilon=0,1085, \tau_{s}=5,34$;

c) $N_{6}, \varepsilon=0,0057, \tau_{s}=189,95$; d) $N_{6}, \varepsilon=0,1492, \tau_{s}=218,82$

We can see on Fig. 4 that the results of calculations of $p(t)$ are close to each other for different $\delta$ (Fig. 4, $a-d$ ). The results are similar to those above (see the table 3 ).

Table 3

The results of calculations for the first group of experiments

\begin{tabular}{|c|c|c|c|c|}
\hline No exp, & Grid & $\delta$ & $\varepsilon$ & $\tau$ \\
\hline 1 & $N_{1}$ & 0,05 & 0,0591 & 5,23 \\
\hline 2 & $N_{1}$ & 0,1 & 0,1085 & 5,34 \\
\hline 3 & $N_{2}$ & 0,05 & 0,0965 & 5,34 \\
\hline 4 & $N_{2}$ & 0,1 & 0,158 & 5,27 \\
\hline 5 & $N_{3}$ & 0,1 & 0,1435 & 4,95 \\
\hline 6 & $N_{6}$ & 0,05 & 0,0904 & 211,76 \\
\hline 7 & $N_{6}$ & 0,1 & 0,1492 & 218,82 \\
\hline 8 & $N_{7}$ & 0,05 & 0,0851 & 221,11 \\
\hline 9 & $N_{7}$ & 0,1 & 0,185 & 214,44 \\
\hline 10 & $N_{8}$ & 0,1 & 0,1301 & 174,98 \\
\hline
\end{tabular}

The use of the grids $N_{6}-N_{10}$ leads to smaller errors (up to 4 times without noise and $\approx$ on 0,02 with noise), but the time of calculations is approximately 20 times more than that for the grids $N_{1}-N_{5}$. We should take into account the fact that the number of nodes in the grids $N_{6}-N_{10}$ is $\approx 3,86$ times more than in the grids $N_{1}-N_{5}$. The change of $\varepsilon_{0}$ as well as $\tau$ leads to smaller time of calculations $\tau_{s}$. The $10 \%$ noise $\delta$ increases errors of calculations from $\approx 8$ times to $\approx 35$ times.

Acknowledgement. This authors were supported by the Science Foundation of Yugra State University (Grant no. 13-01-20/10). 


\section{References}

1. Alifanov O.M. Inverse Heat Transfer Problems. Springer-Verlag, Berlin Heidelberg, 1994, 331 p. DOI: $10.1007 / 978-3-642-76436-3$

2. Kabanikhin S.I. Inverse and Ill-Posed Problems: theory and applications. De Gruyter, Berlin/Boston, 2012, 459 p. DOI: 10.1515/9783110224016

3. Marchuk G.I. Mathematical Models in Environmental Problems. Studies in Mathematics and its Applications. Vol. 16. Elsevier, 1986, 216 p.

4. Orlande H.R.B. Inverse problems in heat transfer: New trends on solution methodologies and applications. Journal of Heat Transfer, 2012, Vol. 134, Issue 3, 031011 (13 pages). DOI: $10.1115 / 1.4005131$

5. Ozisik M.N., Orlando H.A.B. Inverse heat transfer. New-York, Taylor \& Francis, 2000, 352 p.

6. Prilepko A.I., Orlovsky D.G., Vasin I.A. Methods for solving inverse problems in Mathematical Physics. New-York: Marcel Dekker, Inc. 2000, 744 p. DOI: 10.1201/9781482292985

7. Prilepko A.I., Solov'ev V.V. On the solvability of inverse boundary value problems for the determination of the coefficient preceding the lower derivative in a parabolic equation. Differ. Uravn., Vol. 23, no. 1, pp. 136-143.

8. Pyatkov S.G., Goncharenko O.V. Parameter identification and control in heat transfer processes. Bulletin of the South Ural state university. Mathematical modelling, programming and computer software, 2017, Vol. 10, no. 2, pp. 51-62. DOI: 10.14529/mmp170204

9. Pyatkov S.G., Samkov M.L. On some classes of coefficient inverse problems for parabolic systems of equations. Siberian Advances in Mathematics, 2012, Vol. 22, no. 4, pp. 287-302. DOI: $10.3103 / \mathrm{S} 1055134412040050$

10. Pyatkov S.G., Rotko V.V. On some parabolic inverse problems with the pointwise overdetermination. AIP Conference Proceedings, 2017, Vol. 1907, Issue 1, 020008. DOI: 10.1063/1.5012619

11. Dehghan M., Shakeri F. Method of lines solutions of the parabolic inverse problem with an overspecification at a points. Numerical Algorithms, 2009, Vol. 50, no. 4, pp. 417-437. DOI: 10.1007/s11075-008-9234-3

12. Dehghan M. Numerical computation of a control function in a partial differential equation. $A p$ plied mathematics and computation, 2004, Vol. 147, Issue 2, pp. 397-408. DOI: 10.1016/S00963003(02)00733-6

13. Dehghan M. Determination of a control parameter in the two-dimensional diffusion equation. Applied Numerical Mathematics, 2001, Vol. 37, Issue 4, pp. 489-502. DOI: 10.1016/S01689274(00)00057-X

14. Dehghan M. Fourth-order techniques for identifying a control parameter in the parabolic equations. International Journal of Engineering Science, 2002, Vol. 40, Issue 4, pp. 433-447. DOI: $10.1016 / \mathrm{S} 0020-7225(01) 00066-0$

15. Wang S. Numerical solutions to two inverse problems for identifying control parameters in 2dimensional parabolic partial differential equations. American Society of Mechanical Engineers, Heat Transfer Division, HTD, 1992, Vol. 194, pp. 11-16. https://www.scopus.com/inward/record.uri?eid=2s2.0-0027069329\&partnerID=40\&md5=cdb8e3110b98004d91707746c1ead322

16. Wang W., Han B., Yamamoto M. Inverse heat problem of determining time-dependent source parameter in reproducing kernel space. Nonlinear Analysis: Real World Applications, 2013, Vol. 14, no. 1, pp. 875-887. DOI: 10.1016/j.nonrwa.2012.08.009

17. Li Fu-le, Wu Zi-ku, Ye Chao-rong. A finite difference solution to a two-dimensional parabolic inverse problem. Applied Mathematical Modelling, 2012, Vol. 36, Issue 5, pp. 2303-2313. DOI: 10.1016/j.apm.2011.08.025

18. Tatari M., Dehghan M. He's variational iteration method for computing a control parameter in a semi-linear inverse parabolic equation. Chaos, Solitons and Fractals, 2007, Vol. 33, Issue 2, pp. 671677. DOI: 10.1016/j.chaos.2006.01.059

19. Mohebbi A. A numerical algorithm for determination of a control parameter in two-dimensional parabolic inverse problems. Acta Mathematicae Applicatae Sinica. English series, 2015, Vol. 31, Issue 1, pp. 213-224. DOI: 10.1007/s10255-015-0461-9 
20. Vabishchevich P.N., Vasil'ev V.I. Numerically solving the identification problem for the lower coefficient of a parabolic equation. Matematicheskie zametki SVFU (Mathematical notes of NEFU), 2014, Vol. 21, no. 4, pp. 71-87.

21. Triebel H. Interpolation Theory, Function Spaces, Differential Operators. Berlin, VEB Deutscher Verlag der Wissenschaften, 1978, 528 p. DOI: 10.1002/zamm.19790591227

Received August 24, 2018

Bulletin of the South Ural State University Series "Mathematics. Mechanics. Physics" 2018 , vol. 10 , no. 4 , pp. $30-40$

\title{
ОБ ОПРЕДЕЛЕНИИ МЛАДШЕГО КОЭФФИЦИЕНТА В ПАРАБОЛИЧЕСКОМ УРАВНЕНИИ ВТОРОГО ПОРЯДКА
}

\author{
Е.И. Сафонов \\ Югорский государственный университет, г. Ханты-Мансийск, Российская Федерация \\ E-mail: dc.gerz.hd@gmail.com
}

Рассматривается обратная задача восстановления младшего коэффициента, зависящего от времени, в параболическом уравнении второго порядка. Неизвестный коэффициент является управляющим параметром. Обратная задача состоит в нахождении решения начально-краевой задачи для этого параболического уравнения и этого коэффициента зависящего от времени с использованием данных начально-краевой задачи и точечных условий переопределения. Рассмотрены случаи краевых условий Дирихле и условий с косой производной. Описаны условия, при выполнении которых имеет место теорема существования и единственности решений данной обратной задачи, описан метод численного решения и приведено его обоснование. Все рассмотрения проводятся в пространствах Соболева. Решение прямой задачи основано на методе конечных элементов и методе конечных разностей. Предложенный алгоритм численного решения состоит из трех этапов: инициализации массива, описывающего геометрию области и граничного вектора; реализации итерационного расчета искомого коэффициента с использованием метода конечных элементов; реализация метода конечных разностей. Представлены результаты численных экспериментов, построено численное решение модельной обратной задачи в случае краевых условий Неймана, описана зависимость ошибки вычисления управляющего параметра от изменения коэффициентов уравнения и уровня зашумленности данных переопределения для областей с различным количеством узлов, зависящих от расположения точки наблюдения. Результаты вычислений показывают хорошую сходимость метода. В случае введения $10 \%$ случайного шума погрешность между искомым решением и найденным увеличивается от 8 до 35 раз, но график восстановленного коэффициента остается близким к графику решения и повторяет его контуры.

Ключевые слова: метод конечных элементов; параболическое уравнение; обратная задача.

\section{Литература}

1. Alifanov, O.M. Inverse Heat Transfer Problems / O.M. Alifanov. - Springer-Verlag, Berlin Heidelberg, 1994. - $331 \mathrm{p}$.

2. Kabanikhin, S.I. Inverse and Ill-Posed Problems: theory and applications / S.I. Kabanikhin. - De Gruyter, Berlin/Boston, 2012. - 459 p.

3. Marchuk, G.I. Mathematical Models in Environmental Problems. Studies in Mathematics and its Applications / G.I. Marchuk. - Elsevier, 1986. - Vol. 16. - 216 p.

4. Orlande, H.R.B. Inverse problems in heat transfer: New trends on solution methodologies and applications / H.R.B. Orlande // Journal of Heat Transfer. - 2012. - Vol. 134. - Issue 3. - 031011 (13 pages). 


\section{Математика}

5. Ozisik, M.N. Inverse heat transfer / M.N. Ozisik, H.A.B. Orlando. - New-York: Taylor \& Francis, 2000. - $352 \mathrm{p}$

6. Prilepko, A.I. Methods for solving inverse problems in Mathematical Physics / A.I. Prilepko, D.G. Orlovsky, I.A. Vasin. - New-York: Marcel Dekker, Inc., 2000. - 744 p.

7. Прилепко, А.И. О разрешимости обратных краевых задач определения коэффициента перед младшей производной в параболическом уравнении / А.И. Прилепко, В.В. Соловьев // Дифференциальные уравнения. - 1987. - Т. 23, № 1. - С. 136-143.

8. Pyatkov, S.G. Parameter identification and control in heat transfer processes / S.G. Pyatkov, O.V. Goncharenko // Вестник ЮУрГУ. Серия «Математическое моделирование и программирование». - 2017. - Т. 10, № 2. - С. 51-62.

9. Пятков, С.Г. О некоторых классах коэффициентных обратных задач для параболических систем уравнений / С.Г. Пятков, М.Л. Самков // Математические труды. - 2012. - Т. 15, № 1. C. $155-177$.

10. Pyatkov, S.G. On some parabolic inverse problems with the pointwise overdetermination / S.G. Pyatkov, V.V. Rotko // AIP Conference Proceedings. - 2017. - Vol. 1907. - Issue 1. - 020008.

11. Dehghan, M. Method of lines solutions of the parabolic inverse problem with an overspecification at a points / M. Dehghan, F. Shakeri // Numerical Algorithms. - 2009. - Vol. 50, no 4. - P. 417437.

12. Dehghan, M. Numerical computation of a control function in a partial differential equation / M. Dehghan // Applied mathematics and computation. - 2004. - Vol. 147. - Issue 2. - P. 397-408.

13. Dehghan, M. Determination of a control parameter in the two-dimensional diffusion equation / M. Dehghan // Applied Numerical Mathematics. - 2001. - Vol. 37. - Issue 4. - P. 489-502.

14. Dehghan, M. Fourth-order techniques for identifying a control parameter in the parabolic equations / M. Dehghan // International Journal of Engineering Science. - 2002. - Vol. 40. - Issue 4. P. 433-447.

15. Wang, S. Numerical solutions to two inverse problems for identifying control parameters in 2dimensional parabolic partial differential equations / S. Wang // American Society of Mechanical Engineers, Heat Transfer Division, HTD. - 1992. - Vol. 194. - P. 11-16. https://www.scopus.com/inward/record.uri?eid=2-s2.0-0027069329\&partnerID=40\&md5= cdb8e3110b98004d91707746c1ead322

16. Wang, W. Inverse heat problem of determining time-dependent source parameter in reproducing kernel space / W. Wang, B. Han, M. Yamamoto // Nonlinear Analysis: Real World Applications. 2013. - Vol. 14, no. 1. - pp. 875-887.

17. Li Fu-le, Wu Zi-ku, Ye Chao-rong. A finite difference solution to a two-dimensional parabolic inverse problem / Fu-le Li, Zi-ku Wu, Chao-rong Ye // Applied Mathematical Modelling. - 2012. Vol. 36, Issue 5. - pp. 2303-2313.

18. Tatari, M. He's variational iteration method for computing a control parameter in a semi-linear inverse parabolic equation / M. Tatari, M. Dehghan // Chaos, Solitons and Fractals. - 2007. - Vol. 33. Issue 2. - pp. 671-677.

19. Mohebbi, A. A numerical algorithm for determination of a control parameter in twodimensional parabolic inverse problems / A. Mohebbi // Acta Mathematicae Applicatae Sinica. English series. - 2015. - Vol. 31. - Issue 1. - pp. 213-224.

20. Vabishchevich, P.N. Numerically solving the identification problem for the lower coefficient of a parabolic equation / P.N. Vabishchevich, V.I. Vasil'ev // Математические заметки СВФУ. - 2014. T. 21, № 4. - C. 71-87.

21. Triebel, H. Interpolation Theory, Function Spaces, Differential Operators / H. Triebel. - Berlin, VEB Deutscher Verlag der Wissenschaften, 1978. - 528 p.

Поступила в редакцию 24 августа 2018 z. 